\title{
ALGEBRAIC MODELS FOR MEASURE PRESERVING TRANSFORMATIONS
}

\author{
BY \\ N. DINCULEANU AND C. FOIAŞ
}

1. Introduction. The purpose of this paper is to study measure preserving transformations $T$ on probability measure spaces $(X, \Sigma, \mu)$ by means of algebraic models $(\Gamma, U, \varphi)$ (see Definitions 1 and 2).

The results obtained here contain those obtained in [3] concerning algebraic models $(\Gamma, \varphi)$ of measure spaces $(X, \Sigma, \mu)$.

Each transformation possesses algebraic models and conversely every algebraic system is a model for a certain transformation (Theorem 2). Algebraic models determine transformations uniquely up to a conjugacy (Theorem 1).

Transformations with discrete models (see Definition 3) are uniquely determined by $(\Gamma, U)$ (Theorem 3$)$. Such transformations are characterized by the existence of an orthonormal basis $\Gamma^{\prime} \subset L^{2}(\mu)$ of functions $|f| \equiv 1$, which is also a multiplicative group, such that $U_{T} \Gamma^{\prime} \subset C \cdot \Gamma^{\prime}$ (direct product), where $C$ is the circle group (Theorem 5). In certain cases, conjugacy does no more involve $U$ either (Theorem 4). Continuous automorphisms and rotations on an abelian compact group-equipped with Haar measure-are examples of transformations with discrete model (Corollary of Theorem 5), and in fact, every invertible transformation with discrete model is a superposition of an automorphism and a rotation (Theorem 6).

The class of transformations with discrete models contains the transformations with quasi-discrete spectrum (see Abramov [1]) and the transformations with discrete spectrum (see Halmos [5]). Necessary and sufficient conditions are given for algebraic systems in order to be models for transformations with quasi-discrete spectrum (Theorem 10) or with discrete spectrum (Theorem 11). We mention also Theorem 12 which gives necessary and sufficient conditions in order that $\Gamma_{1}=\Gamma_{\infty}$.

In Theorems 7 and 9, ergodicity of transformations is characterized by means of algebraic models.

2. Preliminaries. Let $(X, \Sigma, \mu)$ be a probability measure space and $T: X \rightarrow X$ a measure preserving transformation.

(1) We denote by $\Gamma(\mu)$ the multiplicative group of the (equivalence classes of) functions $f \in L^{\infty}(\mu)$ with $|f| \equiv 1$, by $\varphi_{\mu}$ the function of positive type on $\Gamma(\mu)$ defined by

$$
\varphi_{\mu}(f)=\int f d \mu, \quad \text { for } f \in \Gamma(\mu)
$$

Received by the editors February 26, 1967 and, in revised form, April 12, 1967. 
and by $U_{T}$ the linear isometry defined on $L^{2}(\mu)$ by $U_{T} f=f \circ T$. Then [3, Proposition 1],

$$
\varphi_{\mu}(f)=1 \text { if and only if } f=1
$$

and $U_{T}$ (or, more precisely, the restriction of $U_{T}$ to $\Gamma(\mu)$ ) is an injective homomorphism of $\Gamma(\mu)$ into itself, such that

and

$$
U_{T} c=c, \text { for } c \in C \text { (the circle group) }
$$

$$
\varphi_{\mu}\left(U_{T} f\right)=\varphi_{\mu}(f), \text { for } f \in \Gamma(\mu) .
$$

If $T$ is invertible, then $U_{T}$ is an automorphism of $\Gamma(\mu)$.

(2) For every function $f \in \Gamma(\mu)$ put

$$
w_{T}(f)=U_{T} f \cdot \bar{f} .
$$

Then $w_{T}$ is a homomorphism of $\Gamma(\mu)$ into itself and we have

$$
U_{T} f=w_{T}(f) \cdot f .
$$

$w_{T}(f)$ is called the generalized proper value corresponding to the generalized proper function $f$ of $U_{T}$.

A subgroup $\Gamma \subset \Gamma(\mu)$ is invariant under $U_{T}$ (that is $U_{T} \Gamma \subset \Gamma$ ) if and only if $\Gamma$ is invariant under $w_{T}$ (that is $w_{T} \Gamma \subset \Gamma$ ).

(3) For every integer $n \geqq 0$ put

$$
\Gamma_{n}=\Gamma_{n}(T)=w_{T}^{-n}(C)=\left\{f \in \Gamma(\mu) ; w_{T}^{n}(f) \in C\right\} .
$$

In particular, $\Gamma_{0}=C$ and $\Gamma_{1}$ is the set of the proper functions of $U_{T}$ belonging to $\Gamma(\mu)$. Each $\Gamma_{n}$ is a group invariant under $U_{T}$ and $\Gamma_{n} \subset \Gamma_{n+1}$ for every $n$. The set

$$
\Gamma_{\infty}=\Gamma_{\infty}(T)=\bigcup_{n=0}^{\infty} \Gamma_{n}
$$

is also a subgroup of $\Gamma(\mu)$ invariant under $U_{T}$. Moreover, if $\Gamma \subset \Gamma(\mu)$ is a group such that

$$
C \subset \Gamma \text { and } w_{T}^{-1} \Gamma=\Gamma
$$

then $\Gamma_{\infty} \subset \Gamma$. (In fact, for every $n$ we have $w_{T}^{-n}(C) \subset w_{T}^{-n} \Gamma \subset \Gamma$ ).

In particular, if $\Gamma_{n+1}=\Gamma_{n}$ for some $n$, then $\Gamma_{\infty}=\Gamma_{n}$.

(4) For every integer $k \geqq 0, U_{T}^{k}$ is an injective homomorphism of $\Gamma(\mu)$ into itself and

$$
\varphi_{\mu}\left(U_{T}^{k} f\right)=\varphi_{\mu}(f), \quad \text { for } f \in \Gamma(\mu) .
$$

If $\Gamma \subset \Gamma(\mu)$ is invariant under $U_{T}$, then $\Gamma$ is invariant under $U_{T}^{k}$.

For every $n$ we have

therefore

$$
\Gamma_{n}(T) \subset \Gamma_{n}\left(T^{k}\right)
$$

$$
\Gamma_{\infty}(T) \subset \Gamma_{\infty}\left(T^{k}\right) .
$$


(5) Let $\Gamma$ be an abelian group containing a subgroup $C^{\prime}$ of the circle group $C$, and suppose that $\Gamma=C^{\prime} \cdot \Gamma^{\prime}$ (direct product), where $\Gamma^{\prime}$ is a subgroup of $\Gamma$. Let further $U: \Gamma \rightarrow \Gamma$ be an injective homomorphism such that

$$
U c=c, \text { for } c \in C^{\prime} .
$$

For every $\gamma \in \Gamma^{\prime}$ we have $U_{\gamma} \in \Gamma$, therefore, there exists a number $\rho(\gamma) \in C^{\prime}$ and an element $V_{\gamma} \in \Gamma^{\prime}$ such that

$$
U_{\gamma}=\rho(\gamma) V \gamma .
$$

Then $\rho: \Gamma^{\prime} \rightarrow C^{\prime}$ is a homomorphism and $V: \Gamma^{\prime} \rightarrow \Gamma^{\prime}$ is an injective homomorphism. Moreover, for every $n$ there exists a homomorphism $\rho_{n}: \Gamma^{\prime} \rightarrow C^{\prime}$ such that

$$
U^{n} \gamma=\rho_{n}(\gamma) V^{n} \gamma, \text { for } \gamma \in \Gamma^{\prime} .
$$

In particular, if $\Gamma \subset \Gamma(\mu)$ and $U=U_{T}$, then $V_{\gamma}=\gamma$ and $\rho(\gamma)=w_{T}(\gamma)$, for $\gamma \in \Gamma^{\prime}$ $\cap \Gamma_{1}(T)$.

Conversely, if $\rho: \Gamma^{\prime} \rightarrow C^{\prime}$ is a homomorphism and $V: \Gamma^{\prime} \rightarrow \Gamma^{\prime}$ is an injective homomorphism, then the equality

$$
U(c \gamma)=c \rho(\gamma) V(\gamma), \text { for } c \in C^{\prime} \text { and } \gamma \in \Gamma^{\prime}
$$

defines an injective homomorphism $U: \Gamma \rightarrow \Gamma$ which satisfies

and

$$
U c=c, \quad \text { for } c \in C^{\prime}
$$

$$
U_{\gamma}=\rho(\gamma) V \gamma, \quad \text { for } \gamma \in \Gamma^{\prime} .
$$

(6) Let $\left(X^{\prime}, \Sigma^{\prime}, \mu^{\prime}\right)$ be a probability measure space and $T^{\prime}: X^{\prime} \rightarrow X^{\prime}$ a measure preserving transformation.

The transformations $T$ and $T^{\prime}$ are conjugate (see [5, pp. 44-45]) if there exists a linear isometry

such that

$$
\phi: L^{2}(\mu) \rightarrow L^{2}\left(\mu^{\prime}\right)
$$

and

$$
\begin{aligned}
\phi L^{2}(\mu) & =L^{2}\left(\mu^{\prime}\right), \\
\phi(f g) & =\phi f \cdot \phi g, \quad \text { for } f, g \in L^{\infty}(\mu)
\end{aligned}
$$

$$
\phi U_{T}=U_{T}, \phi .
$$

It follows then that $\phi L^{\infty}(\mu)=L^{\infty}\left(\mu^{\prime}\right)$ and

$$
\|\phi f\|_{\infty}=\|f\|_{\infty}, \text { for } f \in L^{\infty}(\mu) .
$$

ReMARK. To say that $T$ and $T^{\prime}$ are conjugate means that the measures $\mu$ and $\mu^{\prime}$ are conjugate (see [3, Definition 1]) by means of a linear isometry $\phi: L^{2}(\mu) \rightarrow L^{2}\left(\mu^{\prime}\right)$ which satisfies in addition the equality

$$
\phi U_{T}=U_{T^{\prime}} \phi .
$$


The following proposition gives some conjugacy invariants connected to $\Gamma(\mu)$, $U_{T}$ and $\phi_{\mu}$.

Proposition 1. If $T$ and $T^{\prime}$ are conjugate, then there exists an injective homomorphism $\phi: \Gamma(\mu) \rightarrow \Gamma\left(\mu^{\prime}\right)$ having the following properties:

(i) $\phi \Gamma(\mu)=\Gamma\left(\mu^{\prime}\right)$;

(ii) $\phi c=c$, for $c \in C$;

(iii) If $\Gamma \subset \Gamma(\mu)$ generates $L^{2}(\mu)$, then $\phi \Gamma$ generates $L^{2}\left(\mu^{\prime}\right)$;

(iv) If $\Gamma \subset \Gamma(\mu)$ is an orthonormal system in $L^{2}(\mu)$ then $\phi \Gamma$ is orthonormal in $L^{2}\left(\mu^{\prime}\right)$;

(v) $\phi \Gamma_{n}(T)=\Gamma_{n}\left(T^{\prime}\right)$ and $\phi \Gamma_{\infty}(T)=\Gamma_{\infty}\left(T^{\prime}\right)$;

(vi) $\varphi_{\mu}(f)=\varphi_{\mu^{\prime}}(\phi f)$, for $f \in \Gamma(\mu)$;

(vii) $\phi U_{T}=U_{T^{\prime}} \phi$ and $\phi w_{T}=w_{T^{\prime}} \phi$.

In fact, if $\phi$ is a linear isometry of $L^{2}(\mu)$ onto $L^{2}\left(\mu^{\prime}\right)$ realizing the conjugacy between $T$ and $T^{\prime}$, then the restriction of $\phi$ to $\Gamma(\mu)$, still denoted by $\phi$, is the required isomorphism (see also [3, Proposition 2]).

REMARK. We shall see (corollary of Theorem 1) that, conversely, if $\phi$ is an isomorphism of $\Gamma(\mu)$ onto $\Gamma\left(\mu^{\prime}\right)$ satisfying conditions (vi) and (vii), then $T$ and $T^{\prime}$ are conjugate.

3. Algebraic models. The considerations of the preceding section lead to the following

DefINITION 1. A system $(\Gamma, U, \varphi)$ consisting of an abelian group $\Gamma$ with unit 1 , an injective homomorphism $U: \Gamma \rightarrow \Gamma$ and a complex function of positive type $\varphi$ on $\Gamma$ such that $\varphi(\gamma)=1$ if and only if $\gamma=1$ and $\varphi\left(U_{\gamma}\right)=\varphi(\gamma)$, for $\gamma \in \Gamma$, is called an algebraic ergodic system (a.e. system).

Two a.e. systems $(\Gamma, U, \varphi)$ and $\left(\Gamma^{\prime}, U^{\prime}, \varphi^{\prime}\right)$ are said to be isomorphic if there exists an isomorphism $\phi$ of $\Gamma$ onto $\Gamma^{\prime}$ such that

$$
\varphi(\gamma)=\varphi^{\prime}(\phi \gamma), \text { for } \gamma \in \Gamma
$$

and

$$
\phi U=U^{\prime} \phi
$$

If we define the homomorphisms $w: \Gamma \rightarrow \Gamma$ by

$$
w(\gamma)=U \gamma \cdot \gamma^{-1}, \text { for } \gamma \in \Gamma
$$

and the homomorphism $w^{\prime}: \Gamma^{\prime} \rightarrow \Gamma^{\prime}$ in a similar way, then condition $\phi U=U^{\prime} \phi$ above is equivalent to condition $\phi w=w^{\prime} \phi$.

EXAMPLE. If $T$ is a measure preserving transformation on a probability measure space $(X, \Sigma, \mu)$, then $\left(C, U_{T}, \varphi_{\mu}\right)$ and $\left(\Gamma(\mu), U_{T}, \varphi_{\mu}\right)$ are a.e. systems. More generally, for every group $\Gamma \subset \Gamma(\mu)$ invariant under $U_{T},\left(\Gamma, U_{T}, \varphi_{\mu}\right)$ is an a.e. system.

We shall see (Theorem 2 ) that every a.e. system can be obtained in this way. 
RemarKs. $1^{\circ}$. To say that $(\Gamma, U, \varphi)$ is an a.e. system, means that $(\Gamma, \varphi)$ is a measure system (see [3, Definition 2]) and that $U: \Gamma \rightarrow \Gamma$ is an injective homomorphism satisfying $\varphi\left(U_{\gamma}\right)=\varphi(\gamma)$ for $\gamma \in \Gamma$. Then $(\Gamma, \varphi \circ U)$ is also a measure system. Moreover, if $U \Gamma=\Gamma$, then $(\Gamma, \varphi)$ and $(\Gamma, \varphi \circ U)$ are isomorphic measure systems.

Conversely if $(\Gamma, \varphi)$ and $\left(\Gamma, \varphi^{\prime}\right)$ are isomorphic measure systems by means of an isomorphism $U: \Gamma \rightarrow \Gamma$, then $(\Gamma, U, \varphi)$ is an a.e. system and $U \Gamma=\Gamma$.

$2^{\circ}$. To say that two a.e. systems $(\Gamma, U, \varphi)$ and $\left(\Gamma^{\prime}, U^{\prime}, \varphi^{\prime}\right)$ are isomorphic, means that $(\Gamma, \varphi)$ and $\left(\Gamma^{\prime}, \varphi^{\prime}\right)$ are isomorphic measure systems, by means of an isomorphism $\phi: \Gamma \rightarrow \Gamma^{\prime}$ which satisfies $\phi U=U^{\prime} \phi$.

Conversely, if $(\Gamma, \varphi)$ and $\left(\Gamma^{\prime}, \varphi^{\prime}\right)$ are isomorphic measure systems, then taking $U: \Gamma \rightarrow \Gamma$ and $U^{\prime}: \Gamma^{\prime} \rightarrow \Gamma^{\prime}$ the identity mappings, the a.e. systems $(\Gamma, U, \varphi)$ and $\left(\Gamma^{\prime}, U^{\prime}, \varphi^{\prime}\right)$ are isomorphic.

$3^{\circ}$. If $(\Gamma, U, \varphi)$ is an a.e. system, then the set $C^{\prime}=\{\gamma \in \Gamma ;|\varphi(\gamma)|=1\}$ is a group, and $\varphi$ is an injective homomorphism of $C^{\prime}$ into the circle group $C$. If we identify an element $\gamma \in C^{\prime}$ with the number $\varphi(\gamma)=c$, we have (see [3, corollary of Proposition 3])

$$
\varphi(c \gamma)=c \varphi(\gamma), \quad \text { for } c \in C^{\prime} \text { and } \gamma \in \Gamma \text {. }
$$

Moreover,

$$
U c=c, \text { for } c \in C^{\prime} .
$$

In fact, if $c \in C^{\prime}$, then $\varphi(U c)=\varphi(c)=c$, therefore $U c \in C^{\prime}$ and $U c=c$.

If $C^{\prime}$ is divisible, then there exists a group $\Gamma^{\prime} \subset \Gamma$ such that

$$
\Gamma=C^{\prime} \cdot \Gamma^{\prime} \text { (direct product). }
$$

The a.e. system $(\Gamma, U, \varphi)$ can be embedded in an a.e. system $\left(\Gamma_{1}, U_{1}, \varphi_{1}\right)$ such that

$$
\left\{\gamma \in \Gamma_{1} ;\left|\varphi_{1}(\gamma)\right|=1\right\}=C
$$

and then

$$
\Gamma_{1}=C \cdot \Gamma_{1}^{\prime} \text { (direct product). }
$$

In case $U_{\gamma}=\gamma$ (or, equivalently, $w(\gamma)=1$ ) implies $\gamma \in C$, the group $\Gamma^{\prime}$ can be precised:

Proposition 2. Let $(\Gamma, U, \varphi)$ be an a.e. system, let $C^{\prime}=\{\gamma \in \Gamma ; \varphi(\gamma) \in C\}$ and $w(\gamma)=U_{\gamma} \cdot \gamma^{-1}$, for $\gamma \in \Gamma$.

If $C^{\prime}$ is divisible (in particular if $C^{\prime}=C$ ) and if $w(\gamma)=1$ implies $\gamma \in C^{\prime}$, then every injective homomorphism $a \rightarrow \gamma_{a}$ of a group $G \subset w \Gamma$ into $\Gamma$ such that $w\left(\gamma_{a}\right)=a$ for $a \in G$ (in particular the homomorphism $1 \rightarrow \gamma_{1}=1$ of $G=\{1\}$ ) can be extended to an injective homomorphism $a \rightarrow \gamma_{a}$ of $w \Gamma$ into $\Gamma$, such that $w\left(\gamma_{a}\right)=a$, for $a \in w \Gamma$.

If we put $\Gamma^{\prime}=\left\{\gamma_{a} ; a \in w \Gamma\right\}$, then $\Gamma=C^{\prime} \cdot \Gamma^{\prime}($ direct product $)$. 
The proof is similar to that given in $[5, \mathrm{p} .46]$, for ergodic transformations with discrete spectrum.

For every $a \in w \Gamma$ choose $\mu_{a} \in \Gamma$ with $U \mu_{a}=a \mu_{a}$, that is $w\left(\mu_{a}\right)=a$. If $a \in G$ we take $\mu_{a}=\gamma_{a}$. We have

$$
U \mu_{a b}=a b \mu_{a b} \text { and } U \mu_{a} \mu_{b}=a b \mu_{a} \mu_{b}
$$

whence

$$
w\left(\mu_{a b}\right)=w\left(\mu_{a} \mu_{b}\right)=a b .
$$

By hypothesis, there exists a number $\gamma(a, b) \in C^{\prime}$ such that

$$
\mu_{a} \mu_{b}=\gamma(a, b) \mu_{a b} .
$$

If $a, b \in G$, then $\gamma(a, b)=1$. Consider the group $\left\{c \gamma_{a} ; c \in C^{\prime}, a \in G\right\}$ and the homomorphism $p$ of this group into $C^{\prime}$ defined by $p\left(c \gamma_{a}\right)=c$. We have, in particular, $p(c)=c$ for $c \in C^{\prime}$ and $p\left(\gamma_{a}\right)=1$ for $a \in G$. Since $C^{\prime}$ is divisible, $p$ can be extended to a homomorphism, still denoted by $p$, of $w \Gamma$ into $C^{\prime}$.

If we now define

$$
\gamma_{a}=\overline{p\left(\mu_{a}\right)} \mu_{a}, \quad \text { for } a \in w \Gamma
$$

then the requirements of the proposition are fulfilled.

REMARK. Condition: $w(\gamma)=1$ implies $\gamma \in C^{\prime}$, is satisfied, for example, if $U=U_{T}$, where $T$ is an ergodic transformation.

Definition 2. Let $(X, \Sigma, \mu)$ be a probability measure space and $T: X \rightarrow X a$ measure preserving transformation. We say that an a.e. $\operatorname{system}(\Gamma, U, \varphi)$ is an algebraic model of the transformation $T$ if there exists an injective homomorphism $J: \Gamma \rightarrow \Gamma(\mu)$ such that:

(a) $J \Gamma$ generates $L^{2}(\mu)$;

(b) $\varphi(\gamma)=\varphi_{\mu}(J \gamma)$, for $\gamma \in \Gamma$;

(c) $J U=U_{T} J$.

It follows that if $\Gamma \subset \Gamma(\mu)$ is a group generating $L^{2}(\mu)$, and invariant under $U_{T}$, then $\left(\Gamma, U_{T}, \varphi_{\mu}\right)$ is an algebraic model for $T$.

If $(\Gamma, U, \varphi)$ is an algebraic model of $T$ by means of an isomorphism $J$, then, identifying $\Gamma$ and $J \Gamma$ we can consider that $\Gamma \subset \Gamma(\mu), U=U_{T}$ and $\varphi=\varphi_{\mu}$.

If $(\Gamma, U, \varphi)$ is an algebraic model of $T$, then $T$ is invertible (that is $U_{T} L^{2}(\mu)=L^{2}(\mu)$ ) if and only if $U$ is an automorphism of $\Gamma$ (that is $U \Gamma=\Gamma$ ). In particular, a transformation $T$ having $\left(\Gamma_{1}(T), U_{T}, \varphi_{\mu}\right)$ as algebraic model, is always invertible (since $U_{T} \Gamma_{1}=\Gamma_{1}$ ).

REMARK. To say that $(\Gamma, U, \varphi)$ is an algebraic model for $T$ means that $(\Gamma, \varphi)$ is an algebraic model for the measure $\mu$ (see [3, Definition 3]), by means of an isomorphism $J: \Gamma \rightarrow \Gamma(\mu)$ which satisfies, in addition, $J U=U_{T} J$.

Conversely, if $(\Gamma, \varphi)$ is an algebraic model of the measure $\mu$ and if $U: \Gamma \rightarrow \Gamma$ is 
the identity mapping, then $(\Gamma, U, \varphi)$ is an algebraic model for the identity transformation $T: X \rightarrow X$.

Algebraic models determine the transformations uniquely up to a conjugacy:

THEOREM 1. Two measure preserving transformations are conjugate if and only if they possess isomorphic algebraic models.

Let $T$ and $T^{\prime}$ be two measure preserving transformations on the probability measure spaces $(X, \Sigma, \mu)$ respectively $\left(X^{\prime}, \Sigma^{\prime}, \mu^{\prime}\right)$.

If $T$ and $T^{\prime}$ are conjugate, then from Proposition 1 we deduce that their algebraic models $\left(\Gamma(\mu), U_{T}, \varphi_{\mu}\right)$ and $\left(\Gamma\left(\mu^{\prime}\right), U_{T^{\prime}}, \varphi_{\mu^{\prime}}\right)$ are isomorphic.

Conversely, suppose that $T$ and $T^{\prime}$ possess isomorphic models $(\Gamma, U, \varphi)$ respectively $\left(\Gamma^{\prime}, U^{\prime}, \varphi^{\prime}\right)$. We may consider $\Gamma \subset \Gamma(\mu), U=U_{T}, \varphi=\varphi_{\mu}$ and $\Gamma^{\prime} \subset \Gamma\left(\mu^{\prime}\right)$, $U^{\prime}=U_{T}^{\prime}$ and $\varphi^{\prime}=\varphi_{\mu^{\prime}}$.

If $\phi$ is an isomorphism of $\Gamma$ onto $\Gamma^{\prime}$ such that

$$
\varphi_{\mu}=\varphi_{\mu^{\prime}} \circ \phi \text { and } \phi U_{T}=U_{T^{\prime}} \phi
$$

then (see [3, Theorem 2]), $\phi$ can be extended to a linear isometry $\phi: L^{2}(\mu) \rightarrow L^{2}\left(\mu^{\prime}\right)$ such that

$$
\phi L^{2}(\mu)=L^{2}\left(\mu^{\prime}\right) \text { and } \phi L^{\infty}(\mu)=L^{\infty}\left(\mu^{\prime}\right)
$$

and

The equality

$$
\phi(f g)=\phi f \cdot \phi g, \quad \text { for } f, g \in L^{\infty}(\mu) .
$$

$$
\phi U_{T} f=U_{T^{\prime}} \phi f, \text { for } f \in \Gamma
$$

remains true first for linear combinations of functions of $\Gamma$ and then for every $f \in L^{2}(\mu)$, so that $T$ and $T^{\prime}$ are conjugate.

COROLlaRY. The transformations $T$ and $T^{\prime}$ are conjugate if and only if the a.e. systems $\left(\Gamma(\mu), U_{T}, \varphi_{\mu}\right)$ and $\left(\Gamma\left(\mu^{\prime}\right), U_{T^{\prime}}, \varphi_{\mu^{\prime}}\right)$ are isomorphic.

The following theorem states that every a.e. system is an algebraic model for some transformation.

THEOREM 2. Every a.e. system $(\Gamma, U, \varphi)$ is an algebraic model for a continuous measure preserving homomorphism $\tau$ on an abelian compact group $G$ equipped with a suitable regular Borel measure $\mu$.

Moreover, if $U \Gamma=\Gamma$, then $\tau$ is an automorphism of $G$.

Consider on $\Gamma$ the discrete topology and take $G=\Gamma^{\wedge}$. Let $\mu$ be the unique regular Borel measure on $G$ such that (Bochner's theorem),

$$
\varphi(\gamma)=\int\langle x, \gamma\rangle d \mu(x), \text { for } \gamma \in \Gamma .
$$


Then the mapping $J: \Gamma \rightarrow \Gamma(\mu)$ defined by

$$
J_{\gamma}=\langle\cdot, \gamma\rangle, \text { for } \gamma \in \Gamma
$$

is an injective homomorphism, $J \Gamma$ generates $L^{2}(\mu)$ and

$$
\varphi(\gamma)=\varphi_{\mu}(J \gamma), \quad \text { for } \gamma \in \Gamma .
$$

We define now the mapping $\tau: G \rightarrow G$ by

$$
\langle\tau x, \gamma\rangle=\left\langle x, U_{\gamma}\right\rangle, \text { for } x \in G \text { and } \gamma \in \Gamma \text {. }
$$

Then $\tau$ is a continuous homomorphism of $G$ into itself, and

$$
J U=U_{\tau} J
$$

If $\Gamma U=\Gamma$, then $\tau$ is injective and $\tau G=G$, therefore $\tau$ is an automorphism of $G$.

It remains to prove that $\tau$ is measure preserving.

Consider the regular Borel measure $\nu$ defined on $G$ by

$$
\nu(A)=\mu\left(\tau^{-1} A\right), \text { for every Borel set } A \subset G .
$$

Then for every $\gamma \in \Gamma$ we have

$$
\varphi(\gamma)=\varphi\left(U_{\gamma}\right)=\int\left\langle x, U_{\gamma}\right\rangle d \mu(x)=\int\langle\tau x, \gamma\rangle d \mu(x)=\int\langle x, \gamma\rangle d \dot{\nu}(x)
$$

By the uniqueness of $\mu$ we deduce that $\mu=\nu$, therefore $\mu\left(\tau^{-1} A\right)=\mu(A)$, for every Borel set $A \subset G$ consequently $\tau$ is measure preserving.

REMARK. The proof of Theorem 2 was used in [4] to prove the following

COROLLARY. Every measure preserving transformation $T$ on a probability measure space $(X, \Sigma, \mu)$ is conjugate to a continuous homomorphism $\tau$ on an abelian compact group $G$ equipped with a suitable regular Borel measure. If $T$ is invertible then $\tau$ is an automorphism of $G$.

\section{Discrete algebraic models.}

DefinItIon 3. An a.e. system $(\Gamma, U, \varphi)$ is said to be discrete if $C \subset \Gamma$ and

$$
\begin{aligned}
\varphi(\gamma) & =\gamma, \text { for } \gamma \in C, \\
& =0, \text { for } \gamma \notin C .
\end{aligned}
$$

REMARKS. $1^{\circ}$. An a.e. system $(\Gamma, U, \varphi)$ is discrete if and only if $\left(\Gamma, U^{n}, \varphi\right)$ is discrete.

$2^{\circ}$. We have

$$
U c=c, \text { and } w(c)=1, \text { for } c \in C
$$

where $w(\gamma)=U_{\gamma} \cdot \gamma^{-1}$ for $\gamma \in \Gamma$ (see Remark 3 after Definition 1). 
$3^{\circ}$. Let $(\Gamma, U, \varphi)$ be an a.e. system with $C \subset \Gamma$. Then $\Gamma=C \cdot \Gamma^{\prime}$ (direct product) where $\Gamma^{\prime}$ is a subgroup of $\Gamma$. To say that $(\Gamma, U, \varphi)$ is discrete, means that

$$
\begin{aligned}
\varphi(\gamma)=1 & \text { for } \gamma=1, \\
& =0 \quad \text { for } \gamma \in \Gamma^{\prime}, \gamma \neq 1 .
\end{aligned}
$$

$4^{\circ}$. Let $(\Gamma, U, \varphi)$ be an a.e. system such that

$$
|\varphi(\gamma)|<1 \text { implies } \varphi(\gamma)=0 .
$$

Then $(\Gamma, U, \varphi)$ is "essentially" a discrete system. In fact we can consider $(\Gamma, U, \varphi)$ as a model of a measure preserving transformation $T$ on a probability measure space, and consider $\Gamma \subset \Gamma(\mu), U=U_{T}$ and $\varphi=\varphi_{\mu}$. Consider then the group $\Gamma_{1}$ $=\{c \gamma ; c \in C, \gamma \in \Gamma\}$; then $\left(\Gamma_{1}, U_{T}, \varphi_{\mu}\right)$ is a discrete model of $T$ and contains the initial model $(\Gamma, U, \varphi)$.

For a discrete system $(\Gamma, U, \varphi)$, the function $\varphi$ is completely determined by $\Gamma$, so that the system itself is completely determined by $(\Gamma, U)$.

Proposition 3. Let $\Gamma$ be an abelian group containing $C$ and let $U: \Gamma \rightarrow \Gamma$ be an injective homomorphism such that

$$
U c=c, \text { for } c \in C .
$$

If we define

$$
\begin{aligned}
\varphi(\gamma) & =\gamma & \text { if } \gamma \in C, \\
& =0 & \text { if } \gamma \notin C,
\end{aligned}
$$

then $(\Gamma, U, \varphi)$ is a discrete system.

In fact, $\varphi$ is of positive type:

$$
\begin{aligned}
\sum_{i, j=1}^{n} \alpha_{i} \bar{\alpha}_{j} \varphi\left(\gamma_{i} \gamma_{j}^{-1}\right) & =\sum_{\gamma_{i} \sim \gamma_{j}} \alpha_{i} \bar{\alpha}_{j} \varphi\left(\gamma_{i} \gamma_{j}^{-1}\right)=\sum_{k} \cdot \sum_{\gamma_{i}, \gamma_{j} \in C \gamma_{k}} \alpha_{i} \bar{\alpha}_{j} \varphi\left(\gamma_{i} \gamma_{j}^{-1}\right) \\
& =\sum_{k} \sum_{\gamma_{i} \in C_{\gamma_{k}}}\left|\alpha_{i} \varphi\left(\gamma_{i} \gamma_{k}^{-1}\right)\right|^{2} \geqq 0
\end{aligned}
$$

where $\gamma_{i} \sim \gamma_{j}$ means $\gamma_{i} \gamma_{j}^{-1} \in C$ and $C \gamma_{k}$ the equivalence classes.

If $\gamma \in C$ then $U_{\gamma}=\gamma$, therefore

$$
\varphi\left(U_{\gamma}\right)=\varphi(\gamma)
$$

if $\gamma \notin C$, then $U_{\gamma} \notin C$ (since $U$ is injective), therefore $\varphi(\gamma)=0$ and $\varphi\left(U_{\gamma}\right)=0$, consequently

$$
\varphi\left(U_{\gamma}\right)=\varphi(\gamma) .
$$

Moreover, $\varphi(\gamma)=1$, if and only if $\gamma=1$, so that $(\Gamma, U, \varphi)$ is a discrete system.

For discrete systems, isomorphism does no more involve functions of positive type. 
THEOREM 3. Two discrete systems $(\Gamma, U, \varphi)$ and $\left(\Gamma^{\prime}, U^{\prime}, \varphi^{\prime}\right)$ are isomorphic if and only if there exists an isomorphism $\phi$ of $\Gamma$ onto $\Gamma^{\prime}$ such that

$$
\phi c=c, \text { for } c \in C
$$

and

$$
\phi U=U^{\prime} \phi .
$$

In fact, if the systems are isomorphic by an isomorphism $\phi$, then for every $c \in C$ we have

therefore $\phi c \in C$, and then

$$
\varphi^{\prime}(\phi c)=\varphi(c)=c \neq 0
$$

$$
\varphi^{\prime}(\phi c)=\phi c
$$

consequently $\phi c=c$.

Conversely, let $\phi: \Gamma \rightarrow \Gamma^{\prime}$ be an isomorphism such that $\phi c=c$ for $c \in C$ and $\phi U=U^{\prime} \phi$. We have to prove that $\varphi=\varphi^{\prime} \circ \phi$. For $c \in C$ we have $\phi c=c$, therefore

$$
\varphi^{\prime}(\phi c)=\phi c=c=\varphi(c) \text {. }
$$

If $\gamma \notin C$, then $\phi \gamma \notin C$ (since $\phi$ is injective), therefore $\varphi^{\prime}(\phi \gamma)=0$ and $\varphi(\gamma)=0$, consequently

$$
\varphi^{\prime}(\phi \gamma)=\varphi(\gamma) .
$$

REMARK. If $(\Gamma, U, \varphi)$ is a discrete system, we shall say also that $(\Gamma, U)$ is a discrete system. If $\left(\Gamma, U_{T}\right)$ is a discrete system and $\Gamma \subset \Gamma(\mu)$, for some transformation $T$ on a measure space $(X, \Sigma, \mu)$, we understand that $\varphi=\varphi_{\mu}$.

From Proposition 3 it follows that $(\Gamma, U)$ is a discrete system provided that $\Gamma$ is an abelian group containing $C$ and $U: \Gamma \rightarrow \Gamma$ is an injective homomorphism such that $U c=c$ for $c \in C$.

For certain discrete models $(\Gamma, U)$ isomorphism does no more involve homomorphisms $U$ either:

THEOREM 4. Let $\left(\Gamma_{1}, U_{1}\right)$ and $\left(\Gamma_{2}, U_{2}\right)$ be two discrete systems and put

$$
w_{i}(\gamma)=U_{i} \gamma \cdot \gamma^{-1}, \text { for } \gamma \in \Gamma_{i}, \quad i=1,2 .
$$

Suppose that

$$
\gamma \in \Gamma_{i} \text { and } w_{i}(\gamma)=1 \quad \text { imply } \gamma \in C, \quad i=1,2 .
$$

Then $\left(\Gamma_{1}, U_{1}\right)$ and $\left(\Gamma_{2}, U_{2}\right)$ are isomorphic, if and only if the groups $w_{1} \Gamma_{1}$ and $w_{2} \Gamma_{2}$ are isomorphic by an isomorphism $\phi$ such that $\phi w_{1}=w_{2} \phi$ and $\phi c=c$ for $c \in C \cap w_{1} \Gamma_{1}$.

If $\left(\Gamma_{1}, U_{1}\right)$ and $\left(\Gamma_{2}, U_{2}\right)$ are isomorphic by means of an isomorphism $\phi: \Gamma_{1} \rightarrow \Gamma_{2}$ such that

$$
\phi U_{1}=U_{2} \phi \text { and } \phi c=c \text { for } c \in C \text {, }
$$

then we have also

$$
\phi w_{1}=w_{2} \phi
$$


From $\phi \Gamma_{1}=\Gamma_{2}$ we deduce then $\phi w_{1} \Gamma_{1}=w_{2} \Gamma_{2}$. The restriction of $\phi$ to $w_{1} \Gamma_{1}$ is the required isomorphism.

Conversely, suppose that $w_{1} \Gamma_{1}$ and $w_{2} \Gamma_{2}$ are isomorphic by means of an isomorphism $\phi: w_{1} \Gamma_{1} \rightarrow w_{2} \Gamma_{2}$ such that $\phi w_{1}=w_{2} \phi$ and $\phi c=c$ for $c \in C \cap w_{1} \Gamma_{1}$.

By Proposition 2 there exists an injective homomorphism $a \rightarrow \gamma_{a}$ of $w_{1} \Gamma_{1}$ into $\Gamma_{1}$ such that $w_{1}\left(\gamma_{a}\right)=a$ for $a \in w_{1} \Gamma_{1}$; then $\Gamma_{1}=C \cdot \Gamma_{1}^{\prime}$ (direct product) where $\Gamma_{1}^{\prime}=\left\{\gamma_{a} ; a \in w_{1} \Gamma_{1}\right\}$.

Consider the groups $G_{1}=w_{1}^{2} \Gamma_{1}$ and $G_{2}=w_{2}^{2} \Gamma_{2}$. Since $\phi w_{1} \Gamma_{1}=w_{2} \Gamma_{2}$ and $\phi w_{1}=w_{2} \phi$ we have $G_{2}=\phi G_{1}$.

If $a \in G_{1}$ then $w_{1}\left(\gamma_{a}\right)=a$ and $a=w_{1}(b)$ for some $b \in w_{1} \Gamma_{1}$, therefore $\gamma_{a}=c b$ for some $c \in C$; if we have also $\gamma_{a}=c_{1} b_{1}$ with $c_{1} \in C$ and $b_{1} \in w_{1} \Gamma_{1}$, then $c \bar{c}_{1}=b_{1} b^{-1}$ $\in w_{1} \Gamma_{1}$, therefore, by hypothesis,

$$
c \bar{c}_{1}=\phi\left(c \bar{c}_{1}\right)=\phi\left(b_{1}\right) \overline{\phi(b)},
$$

whence $c \phi b=c_{1} \phi b_{1}$. We define then unambiguously

$$
\gamma_{\phi a}=c \phi b, \quad \text { if } \gamma_{a}=c b \text { with } c \in C \text { and } b \in w_{1} \Gamma_{1} .
$$

It is easy to see that $\phi a \rightarrow \gamma_{\phi a}$ is an injective homomorphism of $G_{2}$ into $\Gamma_{2}$ such that $w_{2}(\phi a)=\phi a$. By Proposition 2, this homomorphism can be extended to an injective homomorphism $a \rightarrow \gamma_{a}$ of $w_{2} \Gamma_{2}$ into $\Gamma_{2}$ such that $w_{2}\left(\gamma_{a}\right)=a$ for $a \in w_{2} \Gamma_{2}$.

We extend now $\phi$ from $w_{1} \Gamma_{1}$ to $\Gamma_{1}$ by

$$
\psi c \gamma_{a}=c \gamma_{\phi a} \text { for } c \in C \text { and } a \in w_{1} \Gamma_{1} .
$$

$\psi$ is an extension of $\phi$, since if $b \in w_{1} \Gamma_{1}$, then $b=c \gamma_{a}$ for some $c \in C$ and $a \in w_{1} \Gamma_{1}$, whence $a=w_{1}\left(\gamma_{a}\right)=w_{1}(b) \in w_{1}^{2} \Gamma_{1}$ and $\gamma_{a}=\bar{c} b$, therefore $\gamma_{\phi a}=\bar{c} \phi b$; it follows then that $\phi b=c \gamma_{\phi a}=\psi\left(c \gamma_{a}\right)=\psi b$.

Moreover, $\psi$ is an isomorphism of $\Gamma_{1}$ onto $\Gamma_{2}$ and $\psi c=c$ for $c \in C$. Finally, if $c \in C$ and $\gamma_{a} \in \Gamma_{1}^{\prime}$, we have

$$
\psi U_{1} c \gamma_{a}=\psi c a \gamma_{a}=\psi a \cdot \psi c \gamma_{a}=\phi a \cdot c \gamma_{\phi a}=U_{2} c \gamma_{\phi a}=U_{2} \psi c \gamma_{a}
$$

therefore $\psi U_{1}=U \psi_{2}$. By Theorem 3, $\left(\Gamma_{1}, U_{1}\right)$ and $\left(\Gamma_{2}, U_{2}\right)$ are isomorphic.

For transformations with discrete models we have the following characterization:

THEOREM 5. A measure preserving transformation $T$ on a probability measure space $(X, \Sigma, \mu)$ has a discrete model if and only if there exists a set $\Gamma^{\prime} \subset \Gamma(\mu)$ such that

(a) $\Gamma^{\prime}$ is a group;

(b) $\Gamma^{\prime}$ is an orthonormal basis of $L^{2}(\mu)$;

(c) $U_{T} \Gamma^{\prime} \subset C \Gamma^{\prime}$.

We remark first that if $\Gamma^{\prime}$ is a group and an orthonormal basis in $L^{2}(\mu)$, then $\Gamma^{\prime}$ contains no constant function except 1 , so that $C \cdot \Gamma^{\prime}$ is a direct product.

If conditions $a, b$ and $c$ are satisfied, then $\left(C \cdot \Gamma^{\prime}, U_{T}, \varphi_{\mu}\right)$ is a discrete algebraic model for $T$. In fact, $C \subset C \cdot \Gamma^{\prime}$ and $C \cdot \Gamma^{\prime}$ generates $L^{2}(\mu)$; if $c \in C$, then

$$
\varphi_{\mu}(c)=\int c d \mu=c
$$


while if $\gamma \notin C$, then $\gamma=c \cdot \gamma^{\prime}$ for some $c \in C$ and $\gamma^{\prime} \in \Gamma^{\prime}$ with $\gamma^{\prime} \neq 1$, therefore

$$
\varphi_{\mu}(\gamma)=c \int \gamma^{\prime} d \mu=c\left(\gamma^{\prime} \mid 1\right)=0
$$

Conversely, let $(\Gamma, U, \varphi)$ be a discrete algebraic model for $T$; we may suppose $\Gamma \subset \Gamma(\mu), U=U_{T}$ and $\varphi=\varphi_{\mu}$. Write $\Gamma$ as a direct product $\Gamma=C \cdot \Gamma^{\prime}$, where $\Gamma^{\prime}$ is a subgroup of $\Gamma$, containing no constant function except 1. Finally, $\Gamma^{\prime}$ is an orthonormal system, since for $\gamma^{\prime} \in \Gamma^{\prime}$ we have

$$
\begin{aligned}
\int \gamma^{\prime} d \mu=\varphi\left(\gamma^{\prime}\right) & =1 & \text { if } \gamma^{\prime}=1, \\
& =0 & \text { if } \gamma^{\prime} \neq 1 .
\end{aligned}
$$

COROLlARY. If $G$ is an abelian compact group, equipped with Haar measure $\mu$, then continuous automorphisms $\tau^{\prime}$ and rotations $R$ on $G$, as well as their superpositions $\tau=R \tau^{\prime}$, have discrete model.

We remark first that continuous automorphisms $\tau^{\prime}$ and rotations $R$, therefore, their superpositions $\tau=R \tau^{\prime}$, are measure preserving.

The group of characters $\Gamma^{\prime}=G^{\wedge}$ is an orthogonal system in $L^{2}(\mu)$ and $U_{\tau}, \Gamma^{\prime} \subset \Gamma^{\prime}$; if $R$ is defined on $G$ by $R x=c x$, for some $c \in G$, then

$$
U_{\tau} \gamma(x)=\gamma(\tau x)=\gamma(c) \gamma\left(\tau^{\prime} x\right)=\gamma(c) U_{\tau} \gamma(x)
$$

for every $\gamma \in \Gamma^{\prime}$, therefore $U_{\tau} \Gamma^{\prime} \subset C \cdot \Gamma^{\prime}$. By Theorem 5, $\tau$ has discrete model. Conversely:

THEOREM 6. Every invertible measure preserving transformation $T$, with discrete model $\left(\Gamma, U_{T}\right)$, on a probability measure space $(X, \Sigma, \mu)$, is conjugate to the superposition of a continuous automorphism and a rotation on an abelian compact group, equipped with Haar measure.

Consider $\Gamma=C \cdot \Gamma^{\prime}$ (direct product) and

$$
U_{T} \gamma=\rho(\gamma) V \gamma, \text { for } \gamma \in \Gamma^{\prime}
$$

where $\rho$ is a character of $\Gamma^{\prime}$ and $V$ is an injective homomorphism of $\Gamma^{\prime}$. Since $T$ is invertible, we have $U_{T} L^{2}(\mu)=L^{2}(\mu)$, therefore $V \Gamma^{\prime}=\Gamma^{\prime}$. Consider $\Gamma^{\prime}$ endowed with the discrete topology and consider the Haar measure $\nu$ on the abelian compact group $G=\Gamma^{\prime}$. Then $\rho \in G$. We define the continuous homomorphism $\tau^{\prime}$ on $G$ by

$$
\left\langle\tau^{\prime} x, \gamma\right\rangle=\langle x, V \gamma\rangle, \text { for } x \in G \text { and } \gamma \in \Gamma^{\prime} .
$$

Since $V \Gamma^{\prime}=\Gamma^{\prime}, \tau^{\prime}$ is an automorphism. Consider finally the mapping $\tau: G \rightarrow G$ defined by

$$
\tau(x)=\rho \tau^{\prime}(x), \text { for } x \in G
$$


Then $\left(C \cdot G^{\wedge}, U_{\tau}\right)$ is a discrete model for $\tau$, and the mapping $\phi: C \cdot \Gamma^{\prime} \rightarrow C \cdot G^{\wedge}$ defined by

$$
\phi c \gamma=c\langle\cdot, \gamma\rangle, \text { for } c \in C \text { and } \gamma \in \Gamma^{\prime}
$$

is an isomorphism such that $\phi c=c$ for $c \in C$. Moreover, for $\gamma \in \Gamma^{\prime}$ we have

$$
\begin{aligned}
\phi U_{T} \gamma & =\phi \rho(\gamma) V \gamma=\rho(\gamma)\langle\cdot, V \gamma\rangle \\
& =\rho(\gamma)\left\langle\tau^{\prime} \cdot, \gamma\right\rangle=\left\langle\rho \tau^{\prime} \cdot, \gamma\right\rangle=\langle\tau \cdot, \gamma\rangle \\
& =U_{\tau}\langle\cdot, \gamma\rangle=U_{\tau} \phi \gamma
\end{aligned}
$$

and this equality remains valid for $\gamma \in \Gamma$, therefore $\phi U_{T}=U_{\tau} \phi$. By Theorem 3, $T$ and $\tau$ are conjugate.

COROLLARY 1. A measure preserving transformation $T$ on a probability measure space $(X, \Sigma, \mu)$ is conjugate to a continuous automorphism on a compact abelian group, equipped with Haar measure, if and only if there exists a set $\Gamma^{\prime} \subset \Gamma(\mu)$ such that

(a) $\Gamma^{\prime}$ is a group;

(b) $\Gamma^{\prime}$ is an orthonormal basis of $L^{2}(\mu)$;

(c) $U_{T} \Gamma^{\prime}=\Gamma^{\prime}$.

COROLLARY 2. A measure preserving transformation $T$ on a probability measure space $(X, \Sigma, \mu)$ is conjugate to a rotation on an abelian compact group, equipped with Haar measure, if and only if $T$ has a discrete model $\left(\Gamma, U_{T}\right)$ with $\Gamma \subset \Gamma_{1}$.

We mention also the following property of discrete models.

Proposition 4. Let $T$ be a measure preserving transformation on a probability measure space $(X, \Sigma, \mu)$ and let $\left(\Gamma, U_{T}, \varphi_{\mu}\right),\left(\Gamma^{\prime}, U_{T}, \varphi_{\mu}\right)$ be two discrete systems.

If $\left(\Gamma, U_{T}, \varphi_{\mu}\right)$ is a discrete model for $T$ and if $\Gamma \subset \Gamma^{\prime}$, then $\Gamma=\Gamma^{\prime}$.

In fact, let $f \in \Gamma^{\prime}$. If for every $g \in \Gamma$ we had $f g \notin C$, then

$$
\int f g d \mu=0
$$

therefore $f \equiv 0$, which would contradict $|f| \equiv 1$.

It follows that there exists $g \in \Gamma$ with $f g \in C$.

Then $f \in \bar{g} C \subset \Gamma$, therefore $\Gamma^{\prime}=\Gamma$.

5. Ergodic transformations. In this section we give some characterizations of ergodic transformations by means of their algebraic models.

Let $(X, \Sigma, \mu)$ be a probability measure space and $T: X \rightarrow X$ a measure preserving transformation. The transformation $T$ is ergodic if $f \in L^{2}(\mu)$ and $U_{T} f=f$ imply $f=$ constant.

Proposition 5. If $T$ is ergodic, then $\left(\Gamma_{1}(T), U_{T}\right)$ is a discrete system. 
In fact if $\gamma \in \Gamma_{1}(T)-C$, then $U_{T \gamma}=c \gamma$ for some $c \neq 1$ (because $T$ is ergodic), therefore

consequently

$$
\int \gamma d \mu=\int U \gamma d \mu=c \int \gamma d \mu
$$

$$
\int \gamma d \mu=0
$$

REMARKS. $1^{\circ}$. If $T^{n}$ is ergodic for some $n$, then $T$ is ergodic, therefore $\left(\Gamma_{1}(T), U_{T}\right)$ is a discrete system. Theorem 7 below states a somewhat converse property.

$2^{\circ}$. We shall see (Corollary 1 of Proposition 6) that if $T^{n}$ is ergodic for every $n$, then $\left(\Gamma_{\infty}(T), U_{T}\right)$ is a discrete system.

LEMMA. If $T$ has a discrete model $\left(\Gamma, U_{T}\right)$ and if $\Gamma_{1}(T) \subset \Gamma$, then for every natural $n$ we have

$$
\Gamma_{1}\left(T^{n}\right) \cap \Gamma=\Gamma_{1}(T)
$$

Consider $\Gamma=C \cdot \Gamma^{\prime}$, where $\Gamma^{\prime}$ is a group and an orthonormal basis of $L^{2}(\mu)$. Consider the homomorphisms $\rho_{n}: \Gamma^{\prime} \rightarrow C$ and $V: \Gamma^{\prime} \rightarrow \Gamma^{\prime}$ such that $U_{T}^{n} \gamma=$ $\rho_{n}(\gamma) V^{n}(\gamma)$, for $\gamma \in \Gamma^{\prime}$.

Let $\gamma \in \Gamma_{1}\left(T^{n}\right) \cap \Gamma^{\prime}$. Then $U_{T}^{n} \gamma=c \gamma$, for some $c \in C$, therefore $\rho_{n}(\gamma)=c$ and $V^{n} \gamma=\gamma$. Let $k \leqq n$ be the least natural number such that $V^{k} \gamma=\gamma$ and consider the $k$ dimensional space $K$ generated by $\gamma, V \gamma, \ldots, V^{k-1} \gamma$. Then $K$ is invariant under $U_{T}$, therefore there exists a basis $f_{1}, \ldots, f_{k}$ of $K$ consisting of proper functions of $U_{T}$ :

$$
U_{T} f_{i}=c_{i} f_{i}, \quad \text { with } c_{i} \in C \text {. }
$$

Then $f_{i} \in \Gamma_{1}(T) \subset \Gamma$. Moreover, we may take $f_{i} \in \Gamma^{\prime}$ (multiplying each $f_{i}$ by a suitable number of $C$ ). The basis $\left(f_{1}, \ldots, f_{k}\right)$ must then coincide with the basis $\left(\gamma, V \gamma, \ldots, V^{k-1} \gamma\right)$; for example $f_{1}=\gamma$, therefore $U_{T} \gamma=c_{1} \gamma$.

It follows that $\gamma \in \Gamma_{1}(T)$, therefore $\Gamma_{1}\left(T^{n}\right) \cap \Gamma^{\prime} \subset \Gamma_{1}(T)$, consequently $\Gamma_{1}\left(T^{n}\right)$ $\cap \Gamma \subset \Gamma_{1}(T)$.

The converse inclusion follows from $\Gamma_{1}(T) \subset \Gamma_{1}\left(T^{n}\right)$.

THEOREM 7. Suppose that $T$ has a discrete model $\left(\Gamma, U_{T}\right)$ and let $n$ be a natural number. If:

(a) either $\Gamma \subset \Gamma_{1}(T)$, or $\Gamma_{1}(T) \subset \Gamma$;

(b) $\gamma \in \Gamma$ and $U_{T}^{n} \gamma=\gamma$ imply $\gamma \in C$;

then $T^{n}$ is ergodic.

Let $f \in L^{2}(\mu)$ be a function such that $U_{T}^{n} f=f$ and prove that $f$ is constant.

Consider $\Gamma=C \cdot \Gamma^{\prime}$, where $\Gamma^{\prime}$ is a group and an orthonormal basis of $L^{2}(\mu)$. Then

where

$$
f=\sum_{\gamma \in \Gamma^{\prime}} \alpha(\gamma) \gamma
$$

$$
\alpha(\gamma)=\int f \bar{\gamma} d \mu, \quad \text { for every } \gamma \in \Gamma^{\prime}
$$


For every natural number $k \in N$ we have

$$
U_{T}^{k n} f=f
$$

and

$$
U_{T}^{k n} f=\sum_{\gamma \in \Gamma^{\prime}} \alpha(\gamma) \rho_{k n}(\gamma) V^{k n} \gamma
$$

therefore

$$
\alpha\left(V^{k n} \gamma\right)=\alpha(\gamma) \rho_{k n}(\gamma), \quad \text { for } k \in N \text { and } \gamma \in \Gamma^{\prime} \text {, }
$$

whence

$$
\left|\alpha\left(V^{k n} \gamma\right)\right|=|\alpha(\gamma)|, \quad \text { for } k \in N \text { and } \gamma \in \Gamma^{\prime} .
$$

We shall prove that for every element $\gamma \neq 1$ of $\Gamma^{\prime}$ we have $\alpha(\gamma)=0$. Let therefore $\gamma \in \Gamma^{\prime}$ be such that $\gamma \neq 1$.

If $V^{k n} \gamma=\gamma$ for some $k$, then $\gamma \in \Gamma_{1}\left(T^{n}\right)$. In fact, if $\Gamma \subset \Gamma_{1}(T)$, then $\gamma \in \Gamma_{1}\left(T^{n}\right)$ without any other assumption, while if $\Gamma_{1}(T) \subset \Gamma$, then by the preceding lemma

$$
\gamma \in \Gamma_{1}\left(T^{k n}\right) \cap \Gamma=\Gamma_{1}(T)=\Gamma_{1}\left(T^{n}\right) \cap \Gamma .
$$

Writing now the equality $\alpha\left(V^{k n} \gamma\right)=\alpha(\gamma) \rho_{k n}(\gamma)$ for $k=1$ we obtain

$$
\alpha(\gamma)=\alpha(\gamma) \rho_{n}(\gamma)
$$

therefore either $\alpha(\gamma)=0$ or $\rho_{n}(\gamma)=1$. But $\rho_{n}(\gamma)=1$ means $U_{T}^{n} \gamma=\gamma$, which by hypothesis implies $\gamma=1$ and we get a contradiction. It follows that $\alpha(\gamma)=0$.

If $V^{k n} \gamma \neq \gamma$ for every $k$, then the functions $\gamma, V^{n} \gamma, V^{2 n} \gamma$, are different from each other, therefore

$$
\sum_{k=0}^{\infty}\left|\alpha\left(V^{k n} \gamma\right)\right|^{2} \leqq \sum_{\gamma^{\prime} \in \Gamma^{\prime}}\left|\alpha\left(\gamma^{\prime}\right)\right|^{2}<\infty
$$

consequently $\left|\alpha\left(V^{k n} \gamma\right)\right| \rightarrow 0$ as $k \rightarrow \infty$, whence $\alpha(\gamma)=0$.

It follows that $f=\alpha(1) 1$, that is $f$ is constant, consequently $T^{n}$ is ergodic.

RemarKs. $1^{\circ}$. Is it possible to drop condition (a) in the preceding theorem? The answer is positive if condition (b) is satisfied for every $n$ (see Theorem 9 below).

$2^{\circ}$. Is it true that if $T$ is ergodic, then $\Gamma_{1}(T) \subset \Gamma$ for every discrete model $\left(\Gamma, U_{T}\right)$ of $T$ ?

The answer is positive if, in addition, $T^{n}$ is ergodic for every $n$. Moreover, in this case we have $\Gamma_{\infty}(T) \subset \Gamma$ for every discrete model $\left(\Gamma, U_{T}\right)$ of $T$ (see Corollary 2 of Proposition 6).

For ergodic transformations, we have the following conjugacy criterion:

THEOREM 8. Two ergodic transformations $T$ and $T^{\prime}$ with discrete model, are conjugate if and only if there exist discrete models $\left(\Gamma, U_{T}\right)$ and $\left(\Gamma^{\prime}, U_{T^{\prime}}\right)$ of $T$ and $T^{\prime}$ respectively, such that the groups $w_{T} \Gamma$ and $w_{T^{\prime}} \Gamma^{\prime}$ are isomorphic by an isomorphism $\phi$ such that $\phi w_{T}=w_{T}, \phi$ and $\phi c=c$ for $c \in C \cap w_{T} \Gamma$.

We use Theorem 4. 
6. Transformations with ergodic iterates. Let $(X, \Sigma, \mu)$ be a probability measure space and $T: X \rightarrow X$ a measure preserving transformation.

Proposition 6. Suppose that $T^{n}$ is ergodic for every $n$. If $\left(\Gamma, U_{T}\right)$ is a discrete system, then $\left(\bigcup_{n=0}^{\infty} w_{T}^{-n}(\Gamma), U_{T}\right)$ is again a discrete system.

We prove first that $\left(w_{T}^{-1}(\Gamma), U_{T}\right)$ is a discrete system.

It is clear that $w_{T}^{-1}(\Gamma)$ is a subgroup of $\Gamma(\mu)$ invariant under $U_{T}$ and containing $C$. We have to prove that

$$
\varphi_{\mu}(f)=\int f d \mu=0, \text { for } f \in w_{\bar{T}}^{-1}(\Gamma)-C .
$$

Let $f \in w_{T}^{-1}(\Gamma)-C$. There are two possibilities:

(a) $\int U_{T}^{n} f \cdot \bar{f} d \mu=0$, for every $n \geqq 1$.

Then $f, U_{T} f, U_{T}^{2} f, \ldots$ is an orthonormal system in $L^{2}(\mu)$. If $g$ is the projection of 1 on the space generated by this sequence, we have

Then

$$
g=\sum_{n=0}^{\infty} a_{n} U_{T}^{n} f \text { with } \sum\left|a_{n}\right|^{2}<\infty .
$$

$$
\int f d \mu=\int U_{T}^{n} f d \mu=\left(U_{T}^{n} f \mid 1\right)=\left(U_{T}^{n} f \mid g\right)=a_{n}
$$

and $a_{n} \rightarrow 0$, therefore $\int f d \mu=0$.

(b) There exists $n$ such that

Since

$$
\int U_{T}^{n} f \cdot \bar{f} d \mu \neq 0
$$

$$
U_{T}^{n} f \cdot \bar{f}=w_{T}\left(f \cdot U_{T} f \cdot \cdots \cdot U_{T}^{n-1} f\right) \in \Gamma
$$

and since $\left(\Gamma, U_{T}\right)$ is a discrete system, we have

that is

$$
U_{T}^{n} f \cdot \bar{f} \in C
$$

$$
U_{T}^{n} f=c f \text { for some } c \in C .
$$

Since $f$ is not constant and $T^{n}$ is ergodic, we have $c \neq 1$.

Suppose that $n$ is the least natural number satisfying $U_{T}^{n} f=c f$. The $n$-dimensional space $K$ generated by $f, U_{T} f, \ldots, U_{T}^{n-1} f$ is invariant under $U_{T}$, therefore there exists a basis $f_{1}, \ldots, f_{n}$ of $K$ consisting of proper functions of $U_{T}$ :

$$
U_{T} f_{i}=c_{i} f_{i}, \quad \text { with } c_{i} \in C .
$$

Each $f_{i}$ is of the form

therefore

$$
f_{i}=\sum_{k=0}^{n-1} \alpha_{i k} U_{T}^{k f}
$$

$$
U_{T}^{n} f_{i}=c f_{i}
$$


On the other hand

therefore

$$
U_{T}^{n} f_{i}=c_{i}^{n} f_{i}
$$

$$
c_{i}^{n}=c, \text { for every } i
$$

Then $c_{i} \neq 1$ for each $i$ and

$$
\int f_{i} d \mu=\int U_{T} f_{i} d \mu=c_{i} \int f_{i} d \mu
$$

therefore $\int f_{i} d \mu=0$. From

$$
f=\sum_{i=1}^{n} \alpha_{i} f_{i}
$$

we deduce that $\int f d \mu=0$.

By induction we deduce then that for every $n,\left(w_{T}^{-n}(\Gamma), U_{T}\right)$ is a discrete system, therefore $\left(\bigcup_{n=0}^{\infty} w_{T}^{-n}(\Gamma), U_{T}\right)$ is also a discrete system.

COROLLARY 1. If $T^{n}$ is ergodic for every $n$, then $\left(\Gamma_{\infty}(T), U_{T}\right)$ is a discrete system.

In fact $\left(C, U_{T}\right)$ is a discrete system, and $\Gamma_{\infty}(T)=\bigcup_{n=1}^{\infty} w_{T}^{-n}(C)$.

COROLlaRY 2. Suppose that $T$ has a discrete model $\left(\Gamma, U_{T}\right)$. If $T^{n}$ is ergodic for every $n$, then

$$
w_{T}^{-1} \Gamma=\Gamma \text { and } \Gamma_{\infty}(T) \subset \Gamma .
$$

In fact, in this case $\left(w_{T}^{-1} \Gamma, U_{T}\right)$ is again a discrete system and $\Gamma \subset w^{-1} \Gamma$, therefore, by Proposition $3, w^{-1} \Gamma=\Gamma$. Then $\Gamma_{\infty}(T) \subset \Gamma$.

THEOREM 9. Suppose that $T$ has a discrete model $\left(\Gamma, U_{T}\right)$.

If for every natural number $n$, conditions $\gamma \in \Gamma$ and $U^{n} \gamma=\gamma$ imply $\gamma \in C$, then $T^{n}$ is ergodic for every $n$.

Consider $\Gamma$ as a direct product $\Gamma=C \cdot \Gamma^{\prime}$, where $\Gamma^{\prime}$ is a group and an orthonormal basis of $L^{2}(\mu)$.

Consider the homomorphisms $\rho_{n}: \Gamma^{\prime} \rightarrow C$ and $V: \Gamma^{\prime} \rightarrow \Gamma^{\prime}$ such that $U_{T}^{n} \gamma$ $=\rho_{n}(\gamma) V^{n} \gamma$, for $\gamma \in \Gamma^{\prime}$ and $n \in N$.

We shall prove first that $T$ is ergodic. Let $f \in L^{2}(\mu)$ be such that $U_{T} f=f$ and prove that $f$ is constant. We have

$$
f=\sum_{\gamma \in \Gamma^{\prime}} \alpha(\gamma) \gamma
$$

where $\alpha(\gamma)=(f \mid \gamma)$. For every $n$ we have $U_{T}^{n} f=f$ and

therefore

$$
U_{T}^{n} f=\sum_{\gamma \in \Gamma^{\prime}} \alpha(\gamma) \rho_{n}(\gamma) V^{n} \gamma
$$

whence

$$
\alpha\left(V^{n} \gamma\right)=\alpha(\gamma) \rho_{n}(\gamma) \text {, for } \gamma \in \Gamma^{\prime} \text { and } n \in N
$$

$$
\left|\alpha\left(V^{n} \gamma\right)\right|=|\alpha(\gamma)|, \quad \text { for } \gamma \in \Gamma^{\prime} \cdot \text { and } n \in N \text {. }
$$


We shall prove that $\alpha(\gamma)=0$ if $\gamma \neq 1$. We remark that the hypothesis implies that if $\gamma \neq 1$, then $\rho_{n}(\gamma) \neq 1$ for every $n$.

If $\gamma \neq 1$ and $V^{n} \gamma=\gamma$ for some $n$, then the equality $\alpha\left(V^{n} \gamma\right)=\alpha(\gamma) \rho_{n}(\gamma)$ becomes

$$
\alpha(\gamma)=\alpha(\gamma) \rho_{n}(\gamma)
$$

therefore $\alpha(\gamma)=0$.

If $\gamma \neq 1$ and $V^{n} \gamma \neq \gamma$ for every $n$, then the functions $\gamma, V \gamma, V^{2} \gamma, \ldots$ are different from each other, therefore

$$
\sum_{n=1}^{\infty}\left|\alpha\left(V^{n} \gamma\right)\right|^{2} \leqq \sum_{\gamma^{\prime} \in \Gamma^{\prime}}\left|\alpha\left(\gamma^{\prime}\right)\right|^{2}=\|f\|_{2}^{2}<\infty
$$

consequently $\left|\alpha\left(V^{n} \gamma\right)\right|^{2} \rightarrow 0$ as $n \rightarrow \infty$, whence $\alpha(\gamma)=0$.

We deduce that $f=\alpha(1) 1$, that is $f$ is constant, therefore $T$ is ergodic.

We remark now that for every $n,\left(\Gamma, U_{T}^{n}\right)$ is a discrete model for $T^{n}$, satisfying the conditions of the theorem with respect to $U_{T}^{n}$, therefore $T^{n}$ is ergodic.

7. Transformations with quasi-discrete spectrum. Let $(X, \Sigma, \mu)$ be a probability measure space and $T: X \rightarrow X$ a measure preserving transformation.

DEFINITION 4. We say that $T$ has quasi-discrete spectrum if $T$ has a discrete model $\left(\Gamma, U_{T}\right)$ with $\Gamma \subset \Gamma_{\infty}(T)$.

To say that $T$ has quasi-discrete spectrum means that there exists a group $\Gamma^{\prime} \subset \Gamma_{\infty}(T)$ which is an orthonormal basis of $L^{2}(\mu)$, such that $U_{T} \Gamma^{\prime} \subset C \cdot \Gamma^{\prime}$.

Here are some properties of transformations with quasi-discrete spectrum:

(1) If $T$ has quasi-discrete spectrum, then $\left(\Gamma_{\infty}(T), U_{T}, \varphi_{\mu}\right)$ is an (not necessarily discrete) algebraic model of $T$.

(2) If $T$ has quasi-discrete spectrum, then $T^{n}$ has quasi-discrete spectrum, for every $n\left(\right.$ since $\left.\Gamma_{\infty}(T) \subset \Gamma_{\infty}\left(T^{n}\right)\right)$.

(3) If $T$ is conjugate with a transformation with quasi-discrete spectrum, then $T$ has itself quasi-discrete spectrum (see Proposition 1).

(4) Two transformations $T$ and $T^{\prime}$ with quasi-discrete spectrum are conjugate if and only if the a.e. systems $\left(\Gamma_{\infty}(T), U_{T}, \varphi_{\mu}\right)$ and $\left(\Gamma_{\infty}\left(T^{\prime}\right), U_{T^{\prime}}, \varphi_{\mu^{\prime}}\right)$ are isomorphic.

We use Proposition 1 and Theorem 1.

For transformations $T$ for which $\left(\Gamma_{\infty}(T), U_{T}\right)$ is itself a discrete model we have, in addition, the following properties:

(5) Let $T$ and $T^{\prime}$ be two measure preserving transformations having $\left(\Gamma_{\infty}(T), U_{T}\right)$ respectively $\left(\Gamma_{\infty}\left(T^{\prime}\right), U_{T^{\prime}}\right)$ as discrete models.

Then $T$ and $T^{\prime}$ are conjugate if and only if there exists an isomorphism $J$ of $\Gamma_{\infty}(T)$ onto $\Gamma_{\infty}\left(T^{\prime}\right)$ such that

$$
J c=c, \text { for } c \in C
$$

and

$$
J U_{T}=U_{T^{J}} J
$$


(6) If $T$ has quasi-discrete spectrum and if there exists a discrete system $\left(\Gamma^{*}, U_{T}\right)$ with $\Gamma_{\infty}(T) \subset \Gamma^{*}$, then $\left(\Gamma_{\infty}(T), U_{T}\right)$ is a discrete model of $T$ and there is no other discrete model of $T$ containing or contained in $\left(\Gamma_{\infty}(T), U_{T}\right)$.

We use Proposition 3.

For transformations with all iterates ergodic we have some more properties:

(7) If $T^{n}$ is ergodic for every $n$ and if $\Gamma_{\infty}(T)$ generates $L^{2}(\mu)$, then $\left(\Gamma_{\infty}(T), U_{T}\right)$ is a discrete model of $T$ and there is no other discrete model of $T$.

In fact, by Corollary 1 of Proposition $6,\left(\Gamma_{\infty}(T), U_{T}\right)$ is a discrete system, therefore $\left(\Gamma_{\infty}(T), U_{T}\right)$ is a discrete model of $T$. By Corollary 2 of Proposition 6, for any other discrete model $\left(\Gamma, U_{T}\right)$ of $T$ we have $\Gamma_{\infty}(T) \subset \Gamma$, therefore $\Gamma_{\infty}(T)=\Gamma$.

(8) Let $T$ and $T^{\prime}$ be two transformations with quasi-discrete spectrum and all iterates $T^{n}$ and $T^{\prime n}$ ergodic.

Then $T$ and $T^{\prime}$ are conjugate if and only if $w_{T} \Gamma_{\infty}(T)$ and $w_{T^{\prime}} \Gamma_{\infty}\left(T^{\prime}\right)$ are isomorphic by an isomorphism $\phi$ such that $\phi w_{T}=w_{T}, \phi$ and $\phi c=c$ for $c \in C \cap w_{T} \Gamma_{\infty}(T)$.

We use Theorem 8 and property (5) above.

The following theorem gives a characterization of discrete systems which are models for transformations with quasi-discrete spectrum.

THEOREM 10. If $(\Gamma, U)$ is a discrete system such that

$$
\Gamma=\bigcup_{n=0}^{\infty} w^{-n}(C), \text { where } w(\gamma)=U \gamma \cdot \gamma^{-1}, \text { for } \gamma \in \Gamma,
$$

then the corresponding transformation $T$ has quasi-discrete spectrum.

If, in addition, for every natural number $n \in N, \gamma \in \Gamma$ and $U^{n} \gamma=\gamma$ imply $\gamma \in C$, then $T^{n}$ is ergodic for every $n$.

In fact $w$ is the restriction of $w_{T}$ to $\Gamma$, therefore $\Gamma \subset \Gamma_{\infty}(T)$, consequently $T$ has quasi-discrete spectrum.

For the second part of the theorem we use Theorem 9 to deduce that all the iterates $T^{n}$ are ergodic. In this case we have $\Gamma=\Gamma_{\infty}(T)$.

REMARK. Theorem 10 and property (8) were proved by Abramov [1].

Example of transformation with discrete model but without quasi-discrete spectrum . Let $X_{n}=\{-1,1\}$ and $\mu_{n}(\{-1\})=\mu_{n}(\{1\})=\frac{1}{2}$ for $n=0, \pm 1, \pm 2, \ldots$. Consider the product $X=\prod_{n=-\infty}^{\infty} X_{n}$, equipped with the product measure $\mu$ and the bilateral shift $T\left(x_{n}\right)=\left(y_{n}\right)$, where $y_{n}=x_{n+1}$ for every $n$. Then $T^{n}$ is ergodic for every $n$ and the only proper value of $T$ is 1 , so that $w_{T}^{-1}(C)=C$. It follows that $\Gamma_{\infty}(T)=C$ so that $T$ has not quasi-discrete spectrum.

On the other hand, consider the function $f_{0}: X \rightarrow R$ defined by

$$
\begin{aligned}
f_{0}\left(\left(x_{n}\right)\right) & =-1 & & \text { if } x_{0}=-1, \\
& =1 & & \text { if } x_{0}=1,
\end{aligned}
$$

and the group $\Gamma$ generated by $U_{T}^{n} f_{0}, n=0, \pm 1, \pm 2, \ldots$ and by the constants. Then $\left(\Gamma, U_{T}\right)$ is a discrete model of $T$. 
8. Transformations with discrete spectrum. Let $(X, \Sigma, \mu)$ be a probability measure space and $T: X \rightarrow X$ a measure preserving transformation.

DEFINITION 5. We say that $T$ has discrete spectrum if $T$ has a discrete model $\left(\Gamma, U_{T}\right)$ with $\Gamma \subset \Gamma_{1}(T)$.

To say that $T$ has a discrete spectrum means that there exists a group $\Gamma^{\prime} \subset \Gamma_{1}(T)$ of proper functions of $U_{T}$ which is an orthonormal basis of $L^{2}(\mu)$.

Here are some properties of transformations with discrete spectrum:

(1) Every transformation with discrete spectrum is invertible (since $U_{T} \Gamma_{1}=\Gamma_{1}$ ).

(2) A transformation has discrete spectrum if and only if it is conjugate to a rotation on a compact abelian group equipped with Haar measure (see Corollary 2 of Theorem 6).

(3) Every transformation with discrete spectrum has quasi-discrete spectrum.

(4) If $T$ has discrete spectrum, then $\left(\Gamma_{1}(T), U_{T}, \varphi_{\mu}\right)$ is an (not necessarily discrete) algebraic model of $T$.

(5) If $T$ has discrete spectrum, then $T^{n}$ has discrete spectrum for every $n$.

(6) If $T$ is conjugate with a transformation with discrete spectrum, then $T$ has itself discrete spectrum.

(7) Two transformations $T$ and $T^{\prime}$ with discrete spectrum are conjugate if and only if the a.e. systems $\left(\Gamma_{1}(T), U_{T}, \varphi_{\mu}\right)$ and $\left(\Gamma_{1}\left(T^{\prime}\right), U_{T^{\prime}}, \varphi_{\mu^{\prime}}\right)$ are isomorphic.

Transformations $T$ for which $\left(\Gamma_{1}(T), U_{T}\right)$ is itself a discrete model, have additional properties:

(8) Let $T$ and $T^{\prime}$ be two measure preserving transformations having $\left(\Gamma_{1}(T), U_{T}\right)$ respectively $\left(\Gamma_{1}\left(T^{\prime}\right), U_{T^{\prime}}\right)$ as discrete models. Then $T$ and $T^{\prime}$ are conjugate if and only if there exists an isomorphism $J$ of $\Gamma_{1}(T)$ onto $\Gamma_{1}\left(T^{\prime}\right)$ such that:

and

$$
J c=c, \text { for } c \in C
$$

$$
J U_{T}=U_{T^{\prime}} J
$$

(9) If $T$ has discrete spectrum and if there exists a discrete system $\left(\Gamma^{*}, U_{T}\right)$ with $\Gamma_{1}(T) \subset \Gamma^{*}$ then $\left(\Gamma_{1}(T), U_{T}\right)$ is a discrete model of $T$, and there is no other discrete model of $T$ containing or contained in $\left(\Gamma_{1}(T), U_{T}\right)$.

For ergodic transformations we have some more properties:

(10) If $T$ is ergodic and if $\Gamma_{1}(T)$ generates $L^{2}(\mu)$, then $\left(\Gamma_{1}(T), U_{T}\right)$ is a discrete model of $T$, and there is no other discrete model containing or contained in $\left(\Gamma_{1}(T), U_{T}\right)$.

(11) Let $T$ and $T^{\prime}$ be two ergodic transformations with discrete spectrum.

Then $T$ and $T^{\prime}$ are conjugate if and only if $U_{T}$ and $U_{T^{\prime}}$ have the same spectrum $[4$, p. 46].

We use Theorem 8 remarking that $w_{T} \Gamma_{1}(T)$ is the spectrum of $U_{T}$ and $w_{T^{\prime}} \Gamma_{1}\left(T^{\prime}\right)$ is the spectrum of $T^{\prime}$.

The characterization of discrete systems which are models for transformations with discrete spectrum, is given by the following: 
THEOREM 11. If $(\Gamma, U)$ is a discrete system such that

$$
w^{-1}(C)=\Gamma \text {, where } w(\gamma)=U \gamma \cdot \gamma^{-1} \text {, for } \gamma \in \Gamma
$$

then the corresponding transformation $T$ has discrete spectrum.

If, in addition, there exists $n \in N$ such that $\gamma \in \Gamma$ and $U^{n} \gamma=\gamma$ imply $\gamma \in C$, then $T^{n}$ is ergodic.

In fact, $w$ is the restriction of $w_{T}$ to $\Gamma$, therefore $\Gamma \subset \Gamma_{1}(T)$, consequently $T$ has discrete spectrum. The second part follows from Theorem 9 . In this case we have $\Gamma=\Gamma_{1}(T)$.

When does $\Gamma_{1}$ coincide with $\Gamma_{\infty}$ ?

THEOREM 12. Let $T$ be an ergodic transformation with discrete spectrum, on a probability measure space $(X, \Sigma, \mu)$. We have $\Gamma_{1}(T)=\Gamma_{\infty}(T)$ if and only if the point spectrum of $U_{T}$ contains no root of 1 (except 1 itself).

Suppose first that $\Gamma_{1}=\Gamma_{\infty}$. Let $\xi$ be a proper value of a function $f \in \Gamma_{1}$ :

$$
U_{T} f=\xi f \text {. }
$$

We shall prove that if $\xi^{n}=1$ for some $n$, then $\xi=1$. In fact, suppose that $N$ is the least natural number with $\xi^{N}=1$. We have then

$$
U_{T} f^{N}=f^{N}
$$

therefore, (since $T$ is ergodic) $f^{N}$ is constant, and we may suppose that $f^{N} \equiv 1$, multiplying $f$ by a suitable number, if necessary. Then $f$ takes on the values $1, \xi, \ldots$, $\xi^{N-1}$ on the corresponding sets $A_{0}, A_{1}, \ldots, A_{N-1}$

Since $f(T x)=\xi f(x)$, we have

$$
f=\sum_{k=0}^{N-1} \xi^{k} \varphi_{A_{k}}
$$

$$
\sum_{k=0}^{N-1} \xi^{k} \varphi_{T^{-1}} A_{k}=\sum_{k=0}^{N-1} \xi^{k+1} A_{k}
$$

therefore $T A_{k}=A_{k+1}$ for $k=0,1, \ldots, N-1$, where $A_{N}=A_{0}$. It follows that $\mu\left(A_{k}\right)$ $=\mu\left(A_{0}\right)>0$ for every $k$.

If $N$ is odd we take $\nu$ such that $\nu^{N}=1$; if $N$ is even, we take $\nu$ such that $\nu^{N}=-1$.

Define now the function

Then we have

$$
g=\sum_{k=0}^{N-1} \nu^{k} \xi^{k(k-1) / 2} \varphi_{A_{k}}
$$

$$
U g=v f g .
$$

If fact, if $x \in A_{k}$ and $k=0,1, \ldots, N-2$, we have $T x \in A_{k+1}$, therefore

$$
U g(x)=g(T x)=\nu^{k+1} \xi^{k(k+1) / 2}=\nu \xi^{k} \nu^{k} \xi^{k(k-1) / 2}=\nu f(x) g(x)
$$

and for $x \in A_{N-1}$ we have $T x \in A_{N}=A_{0}$, therefore

$$
U g(x)=g(T x)=1=\nu^{N} \xi^{N(N-1) / 2}=\nu \xi^{N-1} \nu^{N-1} \xi^{(N-1)(N-2) / 2}=\nu f(x) g(x)
$$

since $\xi^{N(N-1) / 2}$ is equal to 1 if $N$ is odd and to -1 if $N$ is even. 
It follows that $g \in \Gamma_{2}=\Gamma_{1}$, therefore $\nu f=\alpha \in C$, consequently $f \in C$. Wé deduce then that $U f=f$, therefore $\xi=1$.

Conversely, suppose that $\Gamma_{1} \neq \Gamma_{2}$ and prove that $U_{T}$ has at least a proper value $\alpha \neq 1$ such that $\alpha^{N}=1$ for some $N$.

Let $g_{0} \in \Gamma_{2}-\Gamma_{1}$. Since $\Gamma_{1}$ is an orthonormal basis of $L^{2}(\mu)$ and $g_{0} \neq 0$, there exists $h \in \Gamma_{1}$ such that

$$
\left(g_{0}, \bar{h}\right)=\int g_{0} h d \mu \neq 0
$$

If we put $g=g_{0} h$, we have $g \in \Gamma_{2}-\Gamma_{1}$ and $\int g d \mu \neq 0$. There exists a function $f \in \Gamma_{1}$ such that

$$
U_{T} g=f g .
$$

There exists also a number $\lambda \in C$ such that

$$
U_{T} f=\lambda f \text {. }
$$

By induction, we deduce that for every $n$ we have

$$
U_{T}^{n} g=\lambda^{n(n-1) / 2} f^{n} g .
$$

We have $f^{n} \in C$ for some $n$. In fact, if we had $f^{n} \notin C$ for every $n$, then (since $f^{n} \in \Gamma_{1}$ ),

$$
\int U_{T}^{n} g \bar{g} d \mu=\lambda^{n(n-1) / 2} \int f^{n} d \mu=0
$$

therefore, the sequence $g, U g, U^{2} g, \ldots$ would be orthonormal, consequently

$$
\int g d \mu=\int U_{T}^{n} g d \mu=\left(U_{T}^{n} g, 1\right) \rightarrow 0
$$

and we would get a contradiction.

Let $N$ be the least integer $\geqq 0$ such that

Then

$$
f^{N}=\mu \in C \text {. }
$$

$$
U^{N} g=\lambda^{N(N-1) / 2} \mu g=\xi g .
$$

We have

$$
\xi \int g d \mu=\int U_{T}^{n} g d \mu=\int g d \mu \neq 0
$$

therefore $\xi=1$, consequently

$$
U^{N} g=g .
$$

Since $g$ is not constant and since $\Gamma_{1}$ generates $L^{2}(\mu)$, there exists a proper function $k \neq 1$ of $\Gamma_{1}$ such that $(g, k) \neq 0$. If $\alpha$ is the corresponding proper value:

we have $\alpha \neq 1$ and

$$
U_{T} k=\alpha k
$$

$$
(g, k)=\left(U^{N} g, k\right)=\left(g, U^{-N} k\right)=\left(g, \alpha^{-N} k\right)=\alpha^{N}(g, k)
$$

therefore $\alpha^{N}=1$. 


\section{BIBLIOGRAPHY}

1. L. M. Abramov, Metric automorphisms with quasi-discrete spectrum, Izv. Akad. Nauk SSSR Ser. Mat. 26 (1962), 513-530. (Russian)

2. N. Dinculeanu and C. Foias, A universal model for ergodic transformations on separable measure spaces, Michigan Math. J. 13 (1966), 109-117.

3. - Algebraic models for measures, Illinois J. Math. 12 (1968), 340-351.

4. C. Foiaş, Automorphisms of compact abelian groups, as models for measure-preserving transformations, Michigan Math. J. 13 (1966), 349-352.

5. P. R. Halmos, Lectures on ergodic theory, Publ. Math. Soc. Japan, No. 3, Tokyo, 1956.

6. A. Ionescu Tulcea and C. Ionescu Tulcea, On the lifting property. I, J. Math. Anal. Appl. 3 (1961), 537-546.

7. K. Jacobs, Lecture notes on ergodic theory, Aarhus Universitet, Aarhus, 1962/1963.

\section{UNIVERSITY OF BUCAREST,} BUCAREST, ROMANIA 